\title{
Argentina 2020: vicisitudes y dilemas de la educación pública en contextos de pandemia
}

\begin{abstract}
Resumen
La pandemia Covid-19 y las consecuentes políticas de aislamiento social empujaron a la educación a una virtualización forzosa. Las y los docentes tuvieron que aprender e imaginar formas de utilizar las tecnologías digitales para poder continuar con los procesos de enseñanza-aprendizaje por fuera de la presencialidad. Esto adquirió una complejidad especial en Argentina y Latinoamérica, en un contexto de precarización tanto de la labor docente como de las condiciones de disponibilidad y acceso a las tecnologías digitales y a Internet, indispensable para continuar con la tarea educativa en el contexto actual. Este artículo realiza un repaso por la legislación y las políticas públicas argentinas de la última década, que nos ayudan a comprender los desafíos y dificultades con los que escuelas, alumnado y docentes se vieron enfrentados para continuar con la tarea educativa. Luego, realizamos un análisis de experiencias, percepciones y sentires de docentes que se encontraron ejerciendo la educación virtual / a distancia durante el 2020, recabadas a partir de "Conversaciones en Red I y II", ciclos de debate en línea organizados por la cátedra de Organizaciones Sociales y Educación (Facultad de Ciencias de la Comunicación -UNC) y el Grupo de Trabajo de CLACSO Apropiación de Tecnologías Digitales e Interseccionalidades durante junio, julio y agosto del 2020.
\end{abstract}

Palabras clave: Tecnologías digitales. Educación. Brecha digital. Políticas educativas.

\author{
Susana Morales \\ Universidad Nacional de Córdoba \\ - Argentina \\ susanamorales@unc.edu.ar
}

\footnotetext{
Para citar este artigo:

MORALES, Susana. Argentina 2020: vicisitudes y dilemas de la educación pública en contextos de pandemia.

Revista Linhas. Florianópolis, v. 22, n. 48, p. 10-40, jan./abr. 2021.

DOI: $\mathbf{1 0 . 5 9 6 5 / 1 9 8 4 7 2 3 8 2 2 4 8 2 0 2 1 0 1 0}$

http://dx.doi.org/10.5965/1984723822482021010
} 


\section{Argentina 2020: vicissitudes and dilemmas of public education in pandemic contexts}

\begin{abstract}
The Covid-19 pandemic and the consequent policies of isolation pushed education towards forced virtualization. Teachers had to learn and imagine ways to use digital technologies in order to continue with the teaching-learning processes outside schools. This situation was especially complex in Argentina and Latin America, in a context of precariousness both of the teaching employment and of the conditions of availability and access to digital technologies and the Internet, essential to continue with the educational task in the current context. This article reviews Argentine legislation and public policies of the last decade, which help us understand the challenges and difficulties that schools, students and teachers have faced in order to continue with the educational task. Then, we carried out an analysis of the experiences, perceptions and feelings of teachers who had to exercise virtual / distance education during 2020, collected from "Conversaciones en Red I and II", online debate talks organized by Social Organizations and Education (Faculty of Communication Sciences - National University of Cordoba) and the CLACSO Working Group Appropriation of Digital Technologies and Intersectionalities, during June, July and August 2020.
\end{abstract}

Keywords: Digital technologies. Education. Digital divide. Educational policies.

\section{Argentina 2020: vicissitudes e dilemas da educação pública em contextos de pandemia}

\begin{abstract}
Resumo
A pandemia Covid-19 e as políticas de isolamento consequentes empurraram a educação para a virtualização forçada. Os professores tiveram que aprender e imaginar formas de usar as tecnologias digitais para dar continuidade aos processos de ensino-aprendizagem fora das escolas. Esta situação foi especialmente complexa na Argentina e na América Latina, em um contexto de precariedade tanto do emprego docente como das condições de disponibilidade e acesso às tecnologias digitais e à Internet, essenciais para continuar a tarefa educativa no contexto atual. Este artigo faz uma revisão da legislação e das políticas públicas argentinas da última década, que nos ajudam a compreender os desafios e as dificuldades que escolas, alunos e professores têm enfrentado para dar continuidade à tarefa educacional. Em seguida, realizamos uma análise das experiências, percepções e sentimentos dos professores que tiveram que exercer a educação virtual / a distância durante o ano de 2020, coletadas em "Conversaciones en Red I e II", palestras de debate online organizadas por Organizações Sociais e Educação (Faculdade de Ciências da Comunicação - Universidade Nacional de Córdoba) e Grupo de Trabalho CLACSO Apropriação de Tecnologias Digitais e Interseccionalidades, nos meses de junho, julho e agosto de 2020.
\end{abstract}

Palavras-chave: Tecnologias digitais. Educação. Brecha digital. Políticas educacionais. 
El rojo más puro que existe, por ejemplo, después de haber sido digitalizado se llama así: 11111111000000000000 0000. ¿Por qué hacer algo tan poco poético? Muy sencillo: porque traduciendo un color a un número puedo introducirlo en máquinas que pueden modificarlo, o simplemente transportarlo, o tan solo guardarlo: lo hacen con una irrisoria facilidad, sin margen de error, a una velocidad vertiginosa y con un coste ridículo. Cada vez que quiero ver de nuevo el color real le pido a la máquina que me lo devuelva: y ella lo hace. (BARICCO, 2019, p. 18)

31 de diciembre de 2020. Javier tiene 19 años y cursó un accidentado primer año en la facultad, con clases y evaluaciones online. La pregunta de la noche, minutos después del brindis de comienzo del nuevo año, recibe una respuesta inesperada:

Javier, ¿cuáles son tus deseos para 2021?

Clases presenciales...

Esta expresión condensa varios de los aprendizajes de la pandemia de 2020, y el consiguiente aislamiento (ASPO - Aislamiento Social Preventivo y Obligatorio en Argentina), que paralizó gran parte de las actividades cotidianas en muchos países del mundo. Para el caso de la educación en sus diferentes niveles, la implementación de diversas estrategias de emergencia virtuales no fue suficiente (aunque, seguramente, necesarias).

Aprendimos que la escuela no es un lugar donde solamente se aprende, sino sobre todo donde se debate, se comparte, se recibe y ofrece afecto, amistad, se participa políticamente, se emprenden acciones solidarias, se construyen vínculos.

Aprendimos que lo que se enseña es más profundo y duradero si el aprendizaje se produce colectivamente.

Aprendimos que lo virtual no siempre (ni mucho menos) es lo mejor.

Algunas de estas cuestiones vamos a exponer en el presente artículo, que aborda las reflexiones conjuntas con docentes de diversos niveles y países, fundamentalmente de Argentina, en el marco del Ciclo virtual "Conversaciones en Red", durante los meses que

\footnotetext{
${ }^{1}$ La organización de estos ciclos estuvo a cargo de Susana Morales, Alejandra Restagno y Yanina Arraya.
} 
en 2020 nos mantuvieron lejos de las aulas. Las sesiones se encuentran alojadas en el canal de Youtube de la Facultad de Ciencias de la Comunicación de la UNC - Argentina.

El Ciclo se desarrolló en varias ediciones:

1. “Conversaciones en Red. La virtualización de la educación en contextos de vulnerabilidad socioeducativa"
a. Intercambio de experiencias alternativas (4 de junio).
b. Comunicar y enseñar con herramientas digitales I (9 de junio).
c. ¿Cómo evaluar en contextos de vulnerabilidad? (11 de junio).
d. Comunicar y enseñar con herramientas digitales II (16 de junio).

e. El lugar de lo pedagógico en la virtualidad: enunciaciones desde Latinoamérica (18 de junio).

f. Comunicar y enseñar con herramientas digitales III (23 de junio).

2. “Conversaciones en Red II. Educación a distancia y virtual desde una perspectiva de derechos. Experiencias y debates necesarios"

a. Políticas públicas en educación a distancia/virtual (28 de julio).

b. El trabajo docente en educación virtual/a distancia (30 de julio).

c. Experiencias en educación a distancia: desafíos y aprendizajes (4 de agosto).

d. Tendencias en educación a distancia y virtual (6 de agosto).

e. Tecnología educativa para la educación presencial y virtual (11 de agosto).

f. El futuro de la educación, ¿es la distancia? (13 de agosto). 
En el marco de esos Ciclos de Conversaciones, realizamos una encuesta que fue respondida por 918 personas, algunos de cuyos resultados se exponen en las páginas que siguen.

Este texto analiza la profundidad y el compromiso que caracteriza al campo educativo, ante la urgencia por seguir sosteniendo los espacios de enseñanza-aprendizaje.

\section{Capitalismo de plataforma y educación}

En los pocos años de existencia de las computadoras e Internet, el campo educativo ha transitado un sinuoso camino hacia la virtualización de algunas de sus prácticas. Muchas de ellas han ocurrido en el marco de procesos de privatización y comercialización durante la década de los '9o, recibiendo gran impulso precisamente de la mano de las tecnologías digitales.

Sostiene Adriana Puiggrós, ex Secretaria de Educación de Argentina y una de las analistas más reconocidas de la historia de la educación en ese país:

La educación virtual, hasta no hace muchos meses era sobre todo un objeto del mercado, y las grandes empresas estaban y están (lo subrayo, están) voraces para capturar el mercado de la educación. Pero resulta que, durante la pandemia, la virtualidad incluyó al espacio público, y miles y miles de docentes y alumnos empezaron a aggiornarse en materia de tecnologías, con lo cual tenemos allí un impacto muy grande. (CONVERSACIONES II, 2020a)

Según el primer Censo Nacional del Personal docente y no docente del Sistema Educativo argentino (ARGENTINA, 2014b), había 962.751 personas trabajando en el sistema educativo de educación común (apoyo docente, dirección/gestión, frente a alumnos) y, de acuerdo con datos oficiales de 2017, 11.236.066 estudiantes (de todos los niveles, excepto la educación superior universitaria (ARGENTINA, 2017a). Algo más de 12 millones de personas: para el mercado de las tecnológicas, un mercado tentador.

Pongamos como ejemplo los resultados de la encuesta respondida por quienes participaron del Ciclo de Conversaciones en Red. Cuando consultamos acerca de cuáles 
eran los recursos que habían incorporado en el contexto de la pandemia, las respuestas fueron:

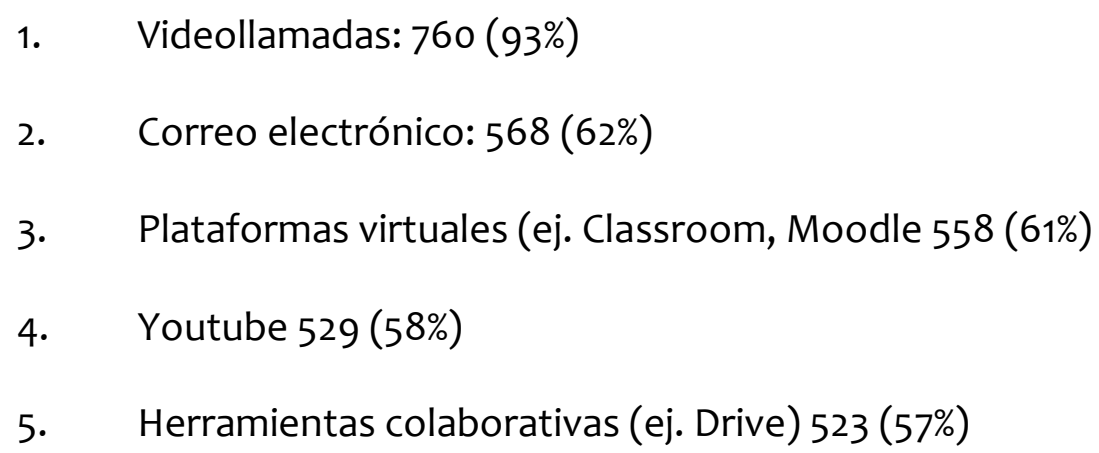

En relación con las videollamadas, las más usadas fueron Facebook, WhatsApp (propiedad de Facebook), Zoom (de la compañía Zoom Video Communications, cuyo creador Eric Yuan figura desde el 2020 entre las 500 personas más ricas del mundo) y Google Meet (propiedad de Google). En cuanto a las herramientas colaborativas, la más utilizada fue Google Drive, también propiedad de Google. Finalmente, en cuanto a las plataformas virtuales, las más recurrentes fueron Google Classroom y Youtube, ambas de Google. Todas estas aplicaciones se ofrecen de manera gratuita través del paquete $G$ Suite for Education, y se articulan entre sí a través de la cuenta de correo Gmail, de... Google. Con lo cual, podemos afirmar que, durante la pandemia, el 100\% de docentes que participaron del Ciclo de Conversaciones en Red y respondieron la encuesta, estuvieron interactuando con aplicaciones de Google para intentar garantizar algún tipo de continuidad educativa de emergencia.

Recordemos que esta empresa posee los siguientes productos (entre otros): cuenta de correo Gmail, buscador Google, Google Maps, Youtube, Google Play, Google Drive, Traductor, Google Imágenes, Google Libros, Google Chrome, Picasa, Google Académico, Android, Chromecast, etc. casi todos ellos utilizados por la mayoría de las personas diariamente.

Cuando en 2011 Siva Vaidhyanathan publicó "La Googlización de todo", ya se imaginaba que - cuando hablaba de todo- era, literalmente, TODO. El autor describe el surgimiento de las principales plataformas y aplicaciones de Google existentes en el 
momento de publicación del libro, y sostiene que el éxito de la empresa se basa en lo que denomina el "sesgo de confianza". Es decir,

sí confiamos a Google nuestra información y preferencias personales, así como nuestro acceso al conocimiento, es porque confiamos en que la tecnología satisface nuestros prejuicios [...] trabaja rápido, produce información que suele parecer relevante y, en consecuencia, parece digno de confianza. (VAIDHYANATHAN, 2012, p. 65)

Esta confianza en las empresas y plataformas digitales, sus aplicaciones y servicios, permea todas nuestras prácticas con tecnologías, y es el fundamento por el cual nos hemos entregado casi sin reparos al uso de tecnologías durante esta pandemia. Sin reparos y sin alternativas.

Según Srnicek (2018), Google y Facebook representaron una vanguardia en un tipo de negocios basado en la extracción de datos como materia prima, el cual caracteriza la etapa actual del capitalismo del siglo XXI, a la que denomina capitalismo de plataformas: "las plataformas se volvieron una manera eficiente de monopolizar, extraer, analizar y usar las cantidades cada vez mayores de datos que se estaban registrando" (SRNICEK, 2018, p. 45). Los datos

educan y dan ventaja competitiva a los algoritmos; habilitan la coordinación y deslocalización de los trabajadores; permiten la optimización y la flexibilidad de los procesos productivos; hacen posible la transformación de productos de bajo margen en servicios de alto margen; y el análisis de datos es en sí mismo generador de datos, en un círculo virtuoso. (SRNICEK, 2018, p. 29)

Por lo cual, no era posible que el sector educativo escapara a esa lógica que adoptó el capitalismo actual para ampliar sus fuentes de acumulación, la tendencia a la plataformización.

Según el Observatorio Latinoamericano de Políticas Educativas (RODRíGUEZ, 2017), en nuestra región -y sobre la base del desprestigio de la educación pública, el ensalzamiento de la educación privada y el desprestigio de los gremios-, la privatización y comercialización de la educación profundizada durante la década de los ‘90, con las 
reformas educativas, implica diversas acciones; por ejemplo, el traspaso de instituciones educativas públicas al sector privado y/o la creación de instituciones educativas de gestión privada, y la contratación por el Estado de servicios de apoyo a lo educativo como seguridad, limpieza y alimentación. En lo que atañe a las tecnologías digitales, las alianzas público-privadas para la capacitación docente y la adquisición y mantenimiento de equipos, programas de software, aplicaciones, elaboración de contenidos y asesorías. Se trata, en definitiva, de "ver al estudiantado y padres de familia como clientes que compran un producto llamado educación, y al cuerpo docente como proveedores de ese servicio y no como educadores" (RODRÍGUEZ, 2017, p. 10).

A poco de declararse la pandemia, en marzo de 2020, la UNESCO puso en marcha una iniciativa global denominada "Coalición Mundial para la Educación en respuesta al COVID 19", para abordar tres ejes fundamentales:

1. Hacer realidad la conectividad universal, para lo cual proponen negociar asociaciones entre los integrantes de la Coalición y proveedores de telecomunicaciones y de Internet. Además, producir investigaciones, incluidos estudios de Big Data para comprender el impacto de los aprendizajes a distancia.

2. Empoderar a los profesores y proteger su estatus, para lo cual se propone brindar capacitación a un millón de docentes para mejorar sus habilidades digitales y pedagógicas, incorporando el aporte de proveedores de MOOC (Massive Open Online Course) y otros actores de la industria de la tecnología educativa. Además, la provisión a docentes de hardware y conectividad gratuita o a bajo costo. Por otro lado, monitoreo y evaluación para medir el progreso realizado por el profesorado e informar de esos progresos a los responsables de la formulación de políticas públicas.

3. Cerrar las desigualdades de género en la educación. En este aspecto, se proponen estudios para analizar el impacto del cierre de las escuelas durante la pandemia. Las propuestas son lo suficientemente imprecisas, como para concluir que lo que no registra adecuadamente la UNESCO es que la inmensa mayoría de docentes son mujeres. Y que la principal desigualdad o brecha de género está dada 
por la cuestión salarial, un tema que está absolutamente fuera de agenda. (UNESCO, 2020)

Según el Observatorio Latinoamericano de Políticas Educativas [OLPE],

en esta Coalición participan ONG's y transnacionales de la tecnología como Microsoft, Google y Facebook, Khan Academy y PRO FUTURO, el proyecto de la Fundación Telefónica, la transnacional de telecomunicaciones Telefónica Movistar. Este llamado de la UNESCO sienta en la mesa de decisiones educativas a las transnacionales de telecomunicaciones y a las corporaciones privadas que lucran con negocios digitales. (OBSERVATORIO LATINOAMERICANO DE POLÍTICAS EDUCATIVAS, 2020a)

Como se aprecia, la carga de la responsabilidad por el sostenimiento del sistema educativo en pandemia sigue recayendo en los colectivos docentes, mientras que los principales beneficiados del sector educativo son los proveedores de infraestructura y conectividad a Internet, de equipamiento/dispositivos, de plataformas y aplicaciones, de contenidos educativos y capacitación. Además de los servicios directos ya mencionados, se benefician con el hecho de que toda práctica de interacción con plataformas digitales por parte de la comunidad educativa, sean o no orientadas a lo educativo, generan datos susceptibles de procesamiento para su comercialización/conversión económica, a los que ya hemos hecho referencia.

\section{Condiciones, disponibilidad y acceso a dispositivos tecnológicos e Internet}

“Esta pandemia ha implicado iluminar las profundas desigualdades que existían antes de la pandemia, particularmente en nuestro continente latinoamericano que sigue siendo el continente más desigual del mundo" (CONVERSACIONES II, 2020c). Con estas palabras comenzaba su intervención el Ministro de Educación de la Argentina en el Ciclo Conversaciones en Red (13 de agosto). En particular, las desigualdades relativas al acceso a dispositivos tecnológicos y a Internet se han expresado, entre otras cuestiones en la imposibilidad para hacer efectivo el contacto entre estudiantes, maestros, maestras, como el acceso a materiales educativos o el envío de las tareas asignadas. 
En mayo de 2020, desde el Grupo de Trabajo de CLACSO Apropiación de Tecnologías Digitales e Interseccionalidades (GT- ATDEI), en conjunto con la Red de Investigadores sobre Apropiación de Tecnologías (RIAT), en el documento "El acceso a las tecnologías digitales como derecho humano", en relación a la virtualización de la educación sosteníamos que "los gobiernos piden continuidad en los procesos pedagógicos en la virtualidad, sin tener en cuenta la inexistencia del acceso a Internet para una parte importante de la población estudiantil, o incluso la no disponibilidad de los dispositivos necesarios" (GT CLACSO, 2020).

Claramente, las políticas públicas de los últimos años han sido insuficientes en este aspecto, lo que muy posiblemente sea de las dos cuestiones más importantes que condicionaron los procesos educativos en el 2020 (la otra cuestión es la de las condiciones laborales de los y las docentes). La puesta en marcha del Programa y Portal Seguimos Educando ${ }^{2}$ para poner a disposición materiales y recursos educativos digitales organizados por nivel y área de temática de conocimiento, se vio limitado en su potencial por esta razón. Es por ello que, de acuerdo a las declaraciones del Ministro de Educación de la Nación Nicolás Trotta

la agenda que desplegamos se relaciona con lo analógico, no solo lo digital. Ya llevamos distribuidos 36 millones de cuadernillos [...] que tiene día una hoja de ruta por día de trabajo entre estudiantes, docentes y familias, y la producción de más de 1.300 horas de producción de televisión y radio. (CONVERSACIONES $I I, 2020 \mathrm{C}$ )

Entonces, para poder comprender qué sucedió durante la pandemia por Covid19 en nuestro país en el campo de la educación, es necesario hacer referencia en primer lugar a este aspecto: la disponibilidad de dispositivos y el acceso a Internet.

Como se sabe, y se ha expuesto en diversos artículos, entre 2010 y 2017 se desplegó en Argentina el Programa Conectar Igualdad (PCI) que procuró equilibrar la brecha digital entre los sectores que podían acceder a los dispositivos y los que no. Las virtudes y limitaciones de ese programa también fueron ya analizadas (véase, por ejemplo:

\footnotetext{
${ }^{2}$ https://www.educ.ar/recursos/150936/seguimos-educando
} 
LARROUQUÉ, 2018; MORALES, 2017; BENÍTEZ LARGHI; WINOCUR, 2016; CASASBLANCAS; CALDEIRO; ODETTI, 2016, LAGO MARTÍNEZ, 2015)33, pero, sin duda, no merecía ser discontinuado como sucedió a poco de asumir el gobierno del Presidente Mauricio Macri en 2015.

Durante los primeros meses de ese gobierno (2015-2016), se produjeron despidos de trabajadores del PCl; en ese momento, el presidente anunció que, a pesar de ello, el programa iba a continuar porque era una política acertada del gobierno anterior (TRAS LOS..., 2016). Sin embargo, en marzo de 2017, por Resolución 1536-E/2017 se dejó sin efecto el Plan Nacional de Inclusión Digital Educativa (aprobado en 2010 y que fuera el marco en el cual nace el Programa Conectar Igualdad), y se creó el Plan Integral Nacional de Educación Digital (PLAINED). Entre sus objetivos se cuentan el de promover la alfabetización digital y el trabajo colaborativo, con énfasis en la programación y la robótica (ARGENTINA, 2017b). Al año siguiente, en abril de 2018, se creó el Plan Aprender Conectados.

¿Cuál fue la evaluación que se hizo desde el gobierno para producir esta reorientación de la política pública en la materia? En el decreto de creación del plan (Resolución 1410/2018), se señala que el 94\% de los docentes cuenta con al menos una computadora en sus hogares, 4 de cada 10 fueron destinatarios de netbooks o notebooks provistas por el Estado y el 98\% de los mismos utiliza teléfono celular (ARGENTINA, 2018a). Por otro lado, el $87,7 \%$ de quienes finalizan la escuela secundaria cuentan con al menos una computadora en sus casas. Por lo tanto, concluyen que el problema de la brecha digital existente en el país, por la cual se creó el Programa Conectar Igualdad, se desplazó hacia la

\footnotetext{
${ }^{3}$ LARROUQUÉ, Damien. El programa Conectar Igualdad en la Argentina (2010-2015). Estado Abierto. Revista Sobre El Estado, La Administración Y Las Políticas Públicas, v. 2, n. 3, p. 117-157, 2018. Disponible en: https://publicaciones.inap.gob.ar/index.php/EA/article/view/63, MORALES, Susana. Prácticas juveniles de apropiación tecno-mediática: qué hacen los estudiantes con las computadoras del Programa Conectar Igualdad. Psicología, Conocimiento y Sociedad, v. 7, n. 2, p. 86-109, 2017. Disponible en: http://revista.psico.edu.uy/., BENÍTEZ LARGHI, Sebastián; WINOCUR, Rosalía. Inclusión digital: una mirada crítica sobre la evaluación del modelo uno a uno en Latinoamérica. Buenos Aires: Teseo, 2016, CASASBLANCAS, Silvina; CALDEIRO, Graciela Paula.; ODETTI, Valeria. La mirada de los sujetos de educación secundaria en los nuevos escenarios educativos. ¿Qué cambió a partir de la llegada de las netbooks de Conectar Igualdad? En: CONGRESO INTERNACIONAL DE EDUCACIÓN DE LA UNIVERSIDAD NACIONAL DE LA PAMPA, 3., 2016, Buenos Aires. Anales [...]. [Buenos Aires]: Pent, 2016. Disponible en: http://www.pent.org.ar/institucional/publicaciones/mirada-sujetos-educac...., LAGO MARTíNEZ, Silvia. De tecnologías digitales, educación formal y políticas públicas. Buenos Aires: Teseo, 2015.
} 
necesidad de la alfabetización digital, ya que "la mera entrega de equipamiento dejó de ser suficiente si no se abordan contenidos específicos con una orientación pedagógica clara e integral en los establecimientos educativos, como núcleos determinantes responsables de los procesos de enseñanza y de aprendizaje" (ARGENTINA, 2018b).

En definitiva, la orientación del gobierno de Macri en este tema estuvo caracterizada por dejar de proveer computadoras a estudiantes y docentes, para proveer a las escuelas de Internet y equipamiento para programación y robótica. El presidente había resumido este pensamiento en una declaración mediática mientras hacía campaña para un segundo período: “¿De qué servía repartir computadoras si las escuelas no tenían conectividad a Internet? Es como repartir asado y no tener parrilla, no tener para prender el fuego" (MAURICIO MACRI, 2019).

Este cambio de rumbo de las políticas se expresa en la ejecución presupuestaria de los diferentes períodos, como se observa en el cuadro 1:

Cuadro 1: Cantidad de computadoras adquiridas en el marco del Programa Conectar Igualdad 4

\begin{tabular}{|l|l|l|l|l|}
\hline 2012 & 2013 & 2014 & 2015 & 2016 \\
\hline 306.828 & 1.624 .959 & 1.011 .991 & 443.192 & 318.231 \\
\hline
\end{tabular}

Fuente: Elaboración propia en base a documentación oficial del Ministerio de Economía de Argentina, 2020.

En 2018, bajo la categoría PLAINED, se destinaron $\$ 3.060,3$ millones, el 71,7\% del total para compra de equipamiento tecnológico y el 19,8\% para Servicios no Personales (gastos de internet y servicios básicos para las instituciones). Esto es, 49.980 equipos para nivel secundario y 1197 para el nivel primario (ARGENTINA, 2018). De 318.231 computadoras portátiles para estudiantes y docentes en 2016, se pasó a 51.177 equipamientos (Aula Digital

\footnotetext{
4 Para los períodos 2017, 2018 y 2019, en el informe de ejecución presupuestaria deja de aparecer el PCl y se incorpora la categoría Educación Digital. Es por ello que no se incorporan los datos de esos períodos. Recordemos que en marzo de 2017 se crea el Plan Nacional Integral de Educación Digital (PLAINED) en reemplazo del Plan Nacional de Inclusión Digital Educativa.
} 
Móvil: un conjunto de dispositivos que se ponen a disposición de los docentes y alumnos para realizar actividades con TIC en las aulas).

El año siguiente, los datos permiten deducir que se adquirieron 15.340 equipos para instituciones de nivel inicial (jardines de infantes) y 15.924 para el nivel primario. Asimismo, se consigna un gasto de $\$ 1.951,0$ millones (más de 1.000 millones menos que el año anterior), que se destinó principalmente a Servicios no Personales (84,9\% del total), en mayor medida correspondiente a gastos en Internet (ARGENTINA, 2019).

En pocos números se pone en evidencia el núcleo de la política de esos años: disminución drástica en la inversión, desaparición de la política de provisión de equipamiento a docentes y estudiantes, provisión de Internet a instituciones (que igualmente resultó insuficiente), focalización en provisión de equipamiento para programación y robótica (en respuesta a las fuertes demandas del mercado de empresas tecnológicas), priorización de niveles inicial y primaria en detrimento de la educación secundaria.

Como lo señala el estudio de CIPPEC (CLAUS; SANCHEZ, 2019), haciendo una comparación de la evolución presupuestaria de los períodos 2015-2019,

las políticas de educación digital y de infraestructura redujeron su volumen promedio de inversión entre ambos períodos, mediando en el primer caso cambios en el modelo de intervención que implicaron menores costos; y en el segundo un cambio de prioridades a favor del nivel inicial, que luego no pudo ejecutarse como se planteó inicialmente. (CLAUS; SANCHEZ, 2019, p.37)

Ese mismo estudio señala que, durante el período 2012-2018 la variación presupuestaria fluctúa entre $\$ 6.694,1$ millones en 2012 y $\$ 1.059,3$ millones en 2018 para los Programas Conectar Igualdad y PLAINED respectivamente (es decir 6 veces menos para este último), tal como se detalla en el gráfico 1: 
Gráfico 1: Presupuesto en educación digital clasificado por inciso (distribución en \%) 20122019

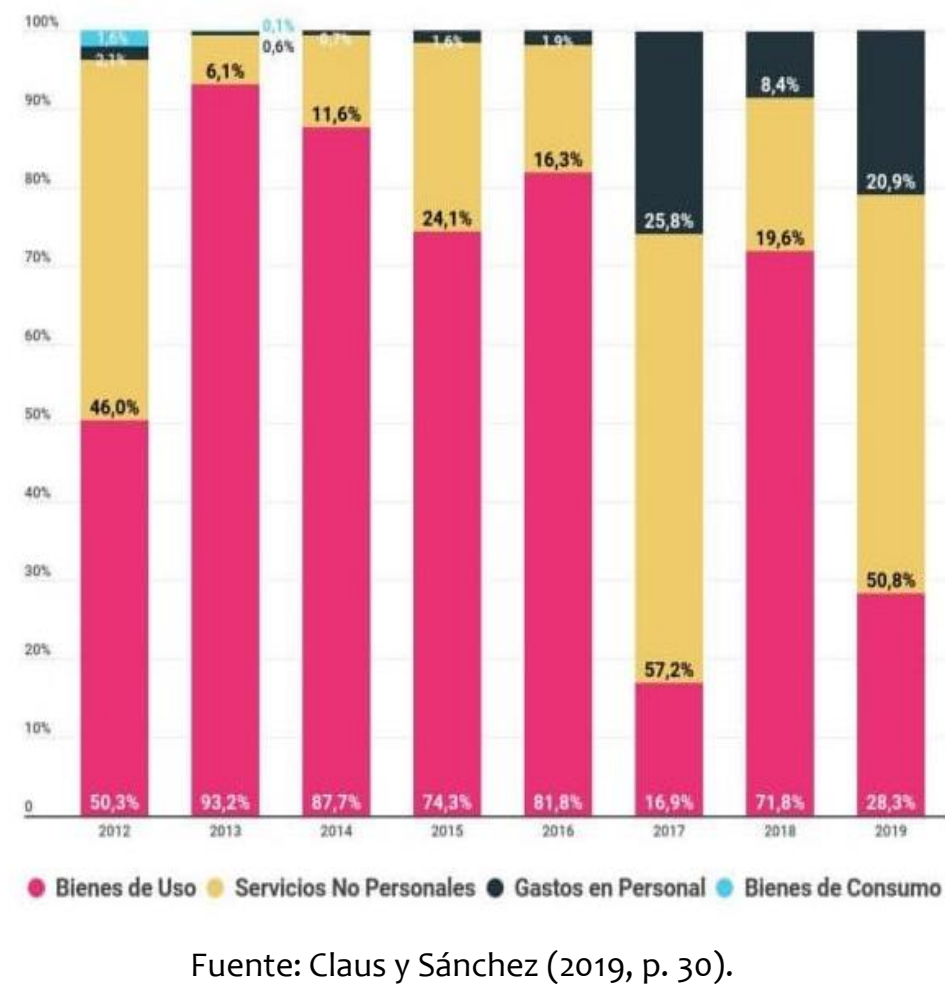

Además, el mismo informe señala que "entre las políticas educativas de mayor peso presupuestario en el ámbito de la cartera educativa nacional se ubican aquellas orientadas a la incorporación de TIC en el sistema educativo y la educación digital en general" (CLAUS; SANCHEZ, 2019, p.29).

De manera que, a la par de haber disminuido el presupuesto con relación al período anterior, estas políticas tuvieron mayor peso que otros rubros educativos como salarios o infraestructura. En 2016 “se devengaron \$1.917,2 millones (100\% del Crédito Vigente) con el objeto de financiar, principalmente, la implementación del sitio web Educ.ar" (ARGENTINA, 2016). Es decir, se trata de un monto de casi el doble de lo que se gastó en 2018 por el PLAINED (1.059,3). Hagamos otra comparación: en el año 2015 el presupuesto devengado por el PCl fue de \$2.352,9 millones (443.192 computadoras). En 2016 fue de \$2.265,2 millones (318.231 computadoras). Como contrapartida, la desproporción con lo que se gastó en la implementación de un sitio web es notable. En ese año también ya se advierte el giro en la política educativa, ya que hubo una asignación presupuestaria vinculada a la 
Provisión de Centros Multimediales para Escuelas de Educación Secundaria, equipándose a 394 escuelas (no se especifica el nivel) (CLAUS; SANCHEZ, 2019).

Al desfinanciamiento en la política de provisión de tecnologías digitales se sumó la torpeza de que muchos equipos disponibles del $\mathrm{PCl}$, no fueron distribuidos sino abandonados en contenedores (TROTTA, 2020).

Ese es el piso desde donde se enfrenta la pandemia 2020 por Covid19, y el consiguiente confinamiento. Según un relevamiento del Observatorio Argentinos por la Educación (LOS CAMBIOS, 2020), casi un 60\% de los estudiantes utilizaron sólo teléfono celular para sus tareas escolares durante la pandemia 2020, dispositivos que en un casi $70 \%$ pertenecían a otro integrante de la familia; es decir, no era propio.

Ahora bien, es de destacar que lo que sucedió en el año 2020 no solamente se trató de la falta de acceso a Internet y dispositivos. La pandemia puso de relieve la importancia de la escuela como espacio físico apto donde se despliegan los procesos educativos. En muchos hogares tampoco se disponía de los espacios físicos, o del ambiente necesario para la concentración y la realización de las tareas escolares. Ello incidió de manera inmediata en la posibilidad de una mínima continuidad educativa por parte de muchos niños, niñas y jóvenes. Así lo expresaba una profesora que participó de nuestros ciclos de conversaciones:

Mi escuela pidió enviar 11 trabajos consecutivos por cada materia a alumnos de zona vulnerada, de asentamientos de villa. Los chicos no tienen conexión, no tienen suficientes celulares, están con problemas económicos en contextos en donde hoy casi no tienen cómo subsistir las familias, y la escuela los tapó de trabajos antes de preguntarles cómo estaban sus familias o cómo estaban ellos. (CONVERSACIONES I, 2020b)

Esta situación fue particularmente grave en los contextos rurales: “Mis estudiantes son de zona rural, sin conectividad, sin herramientas tecnológicas, y en algunos casos sin electricidad. Imposible una interacción, un diálogo. Se envía la actividad y la devuelven cuando pueden" (CONVERSACIONES I, 2020C).

Por otro lado, si bien la situación de precariedad en las condiciones de vida del alumnado en épocas "normales" no pasa inadvertida para gran parte de las y los docentes, 
se produjo un reconocimiento de otro tipo, de detalles de sus vidas que en el devenir de las rutinas escolares ordinarias no se conocen, y ello significó para algunos un tipo de shock particular: "No se visibilizaban las desigualdades... ¿era necesario esto para ver el mundo dolorido de muchos de nuestros estudiantes?", señalaba una profesora (CONVERSACIONES I, 2020d). Y también: “Creo que esta pandemia hizo que más de uno tenga que llegar hacia los alumnos, su contexto, sus realidades, alumnos que trabajan para sostener a sus familias, sabiendo a qué se exponen..." (CONVERSACIONES I, 2020d).

De manera que, en muchas ocasiones, más que proveer educación estrictamente, la tarea docente se complementó o se concentró en contener, apoyar y proveer en relación con otras necesidades básicas del estudiantado.

Asimismo, también quedaron expuestas las dificultades para el acceso por parte de muchos profesores y profesoras. En la encuesta realizada, encontramos que el $99 \%$ de los docentes posee teléfono celular. Esos porcentajes disminuyen cuando se trata de otras tecnologías, como observamos en el gráfico 2:

Gráfico 2: Disponibilidad de equipamiento tecnológico por parte de docentes

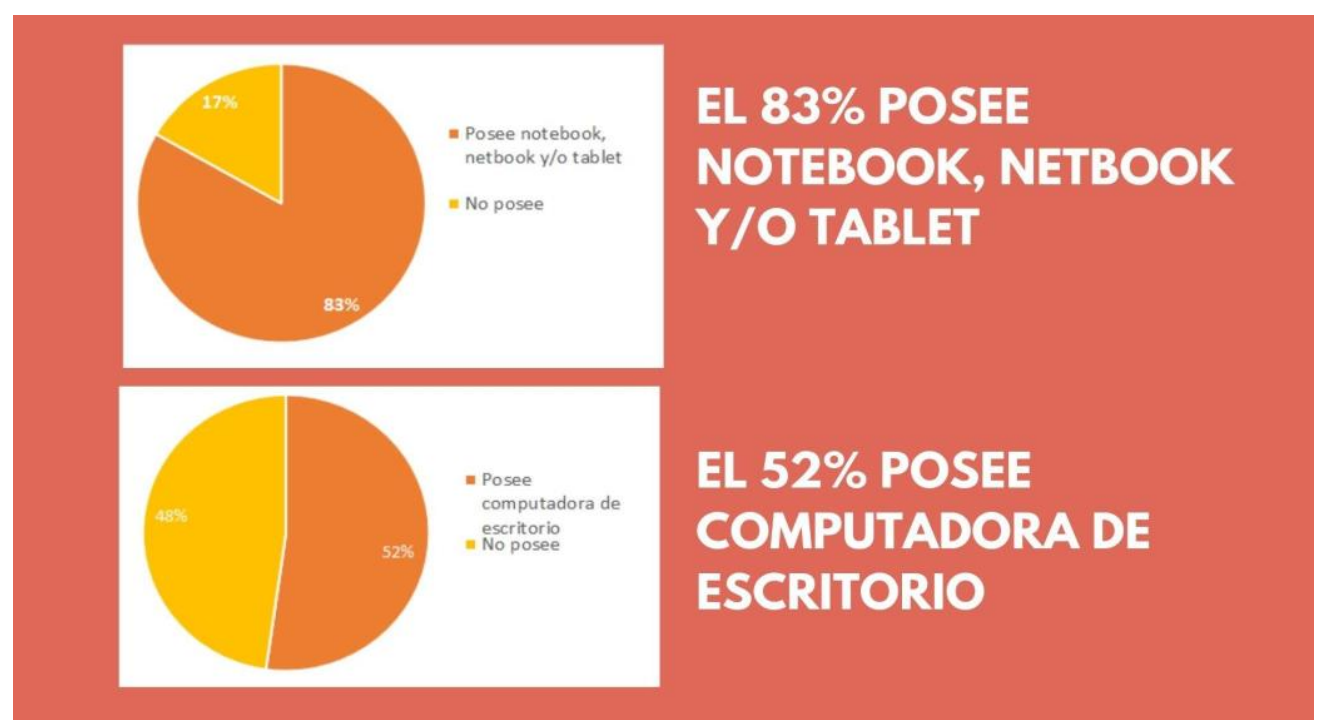

Fuente: Elaboración propia a partir de encuesta realizada a participantes del Ciclo Conversaciones en Red, 2020.

Por último, cuando indagamos acerca de la conectividad a Internet, la situación es aún más desfavorable, de acuerdo con lo que refleja el gráfico 3: 
Gráfico 3: Acceso a Internet en los hogares de docentes

\section{¿POSEE EN SU HOGAR UNA BUENA CONEXIÓN A INTERNET?}

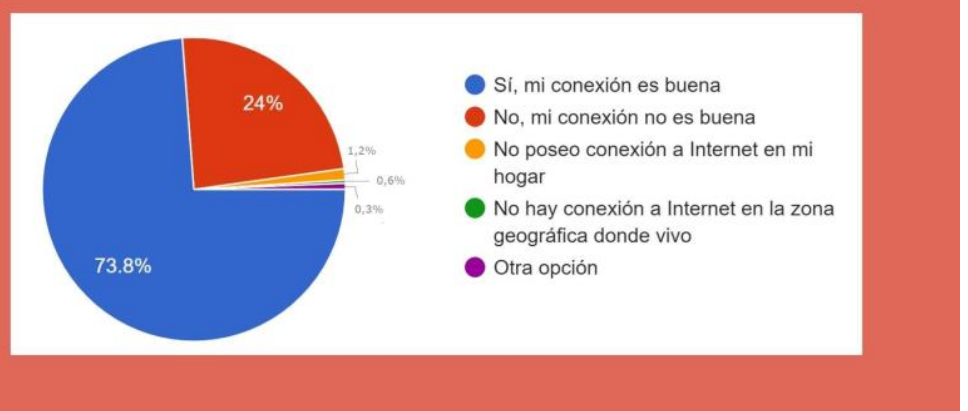

Fuente: Elaboración propia a partir de encuesta realizada a participantes del Ciclo Conversaciones en Red, 2020.

Es decir, un alto porcentaje del profesorado no posee una buena conexión a Internet, o directamente no la posee, y en esas condiciones debió afrontar la tarea educativa en pandemia, de manera $100 \%$ virtual.

Con el correr de los meses, en algún sentido y como consecuencia esperable devenida de estas dificultades, se produjo un desplazamiento en el esfuerzo educativo de docentes: si en los primeros meses de la pandemia/cuarentena ese esfuerzo estuvo focalizado en los procesos de aprendizaje, en una segunda etapa, y habiéndose verificado una mayor pérdida de los contactos y vínculos entre estudiantes y la institución escolar, se orientó a recuperar los vínculos, a retomar los contactos: "a nosotros los docentes, nos hizo repensar... ¿cómo llegar a los que no pueden conectarse, que lamentablemente quedan excluidos?, debemos, como docentes, insertarlos nuevamente..." (CONVERSACIONES I, 2020d).

Tarea sin duda difícil si se tiene en cuenta que, en relación al acceso a Internet, alrededor del 40\% de los estudiantes durante la pandemia sólo accedió a través de datos prepagos o con abono desde el celular. Es decir, no poseía conectividad wi-fi o banda ancha en el hogar. 
La realidad se impuso, e interpeló a docentes haciéndoles voltear la mirada hacia otros ángulos de la experiencia educativa, lo que se refleja en los siguientes testimonios volcados en el chat y en las exposiciones del ciclo Conversaciones en Red:

“En la escuela encuentran afecto, olvidarse de la realidad en la que se encuentran, pero sobre todo socializar, que es necesario para desarrollarse como persona, el compartir, el encuentro con los amigos, la convivencia" (CONVERSACIONES I, 2020C).

“En mi caso soy docente de inglés en primaria, y ahí nos posicionamos en valorizar el vínculo docente-estudiante-familia. Priorizar el proceso más vincular (contención, escucha, acompañamiento en el manejo de las tecnologías), más que evaluar con notas y puntajes".

"Nos interpela a pensar en otros modos de vincularnos los docentes y los estudiantes. Hoy más que nunca fue necesario el ¿cómo estás?, para luego pensar en lo académico" (CONVERSACIONES I, 2020C).

“Trabajamos con la coordinadora y una profe que nos ayuda con el centro de estudiantes a darles ánimos, a ver si siguen adelante, detectar si hay algún otro problema, porque por ahí cuando no tenemos la comunicación personal no nos damos cuenta de qué es lo que le pasa al otro" (CONVERSACIONES I, 2020a).

Como lo señalaba Adriana Puiggrós ${ }^{5}$ en nuestro Ciclo de Conversaciones en Red (en la Sesión del 28 de julio), si uno de los problemas estructurales históricos de la educación en América Latina era el abandono y la deserción, originada en la desconexión educadoreducando, con la pandemia este proceso de desconexión forzosa de grandes sectores de la población con la escuela y con los docentes se profundizó, en tanto que "la reconexión de alguien que ha quedado desconectado es muy difícil física y psicológicamente" (CONVERSACIONES II, 2020a).

\footnotetext{
${ }^{5}$ En el momento de esa disertación se desempeñaba como Secretaria de Educación de la Nación, cargo al que renunció el 19 de agosto de 2020.
} 
Todas estas situaciones de dificultades o directamente falta de acceso a las tecnologías e Internet, se fueron haciendo más agudas y escasamente registradas por las autoridades nacionales y jurisdiccionales de educación actuales.

En los primeros meses de 2020, desde el Ministerio de Educación del nuevo gobierno de Alberto Fernández, se anunció que el $\mathrm{PCl}$ iba a ser reimplementado. Posiblemente sea una promesa que hubiera sido cumplida y que la pandemia dejó trunca. Sin embargo, un gobierno que había comenzado su gestión sobre el final de 2019, habiendo transcurrido más de 5 meses de confinamiento, aún no había demostrado capacidad de reacción en este aspecto. A tal punto que algunas organizaciones sociales alzaron sus voces de manera más contundente.

El 11 de agosto, la organización La Garganta Poderosa publicó un posteo en sus redes titulado "Y después, nos dicen maleducados". Luego de reprochar la falta de respuestas del gobierno ante las graves carencias en materia de conectividad, lo que repercute en la falta de continuidad educativa mínima, señalan que "aquí estamos, sin Historia, ni Matemáticas, ni Biología, pero con lengua todavía, al menos para pegar un grito de corazón... ¡NO TENEMOS MINISTERIO DE EDUCACIÓN!”. Así, anunciaban la propuesta de una campaña propia denominada \#ContagiáConectividad. La foto que ilustra el posteo es un cuaderno escrito con la frase "SIN INTERNET NO SE PUEDE ESTUDIAR" (LA GARGANTA PODEROSA, 2020).

El 22 de agosto, diez días después de esa publicación, se dio a conocer el Decreto 690/2020. El mismo implica una modificación de algunos artículos de la Ley N. ${ }^{\circ} 27.078$ Argentina Digital (2014a), y la incorporación de nuevas normativas. Particularmente se establece que "Ios Servicios de las Tecnologías de la Información y las Comunicaciones (TIC) y el acceso a las redes de telecomunicaciones para y entre licenciatarios y licenciatarias de servicios TIC son servicios públicos esenciales y estratégicos en competencia" (ARGENTINA, 2020b, Art. 1). Esto implicó, como también lo estableció el decreto, que los aumentos de estos servicios quedaran suspendidos hasta el 31 de diciembre de 2020. Por otro lado, el decreto estableció que "la autoridad de aplicación establecerá en la reglamentación la prestación básica universal obligatoria que deberá ser brindada en condiciones de igualdad" (ARGENTINA, 2020b, Art. 3). Esta Prestación Básica 
Universal fue anunciada a mediados de diciembre, para entrar en vigencia a partir del 1 de enero de 2021, consistente en tarifas mínimas para cada servicio de telefonía móvil, paquete de datos de Internet, telefonía fija, acceso a Internet domiciliaria y televisión por suscripción. Las personas beneficiarias son fundamentalmente quienes no poseen ingresos para proveerse los servicios de otro modo, pero también pueden acceder clubes de barrio y entidades de bien público.

Políticas necesarias pero demoradas, cuya eficacia deberá analizarse oportunamente. Si no se entiende que la conectividad y disponibilidad de equipamiento se han transformado en un derecho que permite la adquisición de otros derechos, se terminan profundizando las inequidades. Como también lo anticipamos desde el GT CLACSO ATDEIRIAT,

a las diferencias en los aprendizajes a nivel de las habilidades, la autorregulación, la planificación de los tiempos y la metacognición desafíos que aún persisten en la educación - se suman las experiencias de acceso y producción desiguales entre las y los estudiantes, lo que condiciona una formación crítica y reflexiva en el contexto de las particularidades que se imponen a la educación en tiempos de pandemia. (GT CLACSO, 2020)

Esto es precisamente lo que expresaba una docente durante nuestras Conversaciones en Red:

Va más allá de nuestras propias destrezas y de toda la creatividad que pongamos en nuestras prácticas. Intentamos el año anterior empezar con classroom, los docentes apoyaron muy poco, los alumnos no podían crear ni recordar sus propios mails, llevó meses y el intento naufragó. Cuando este año necesitamos usar la herramienta, no se recuperó la experiencia previa. Alumnos que están llegando a primer año del secundario sin leer ni escribir, están excluidos no sólo por la falta de acceso tecnológico, sino de habilidades y competencias que no han podido alcanzar. El Estado no los visibilizaba entonces. Hoy requerimos ciertas competencias para enseñar en la virtualidad que no fueron brindadas porque las políticas no quisieron ver que nuestros alumnos, en la villa (barrios muy pobres), no leen, no escriben, no comprenden y su autonomía de trabajo es mínima. (CONVERSACIONES I, 2020d) 


\section{Condiciones de trabajo y formación docente}

Como lo señalamos anteriormente, otro de los aspectos que fueron críticos en el proceso de virtualización educativa fueron las condiciones de trabajo del profesorado.

Tal como lo señalaba en la Conversación del 30 de julio Javier Blanco, dirigente de ADIUC (gremio docente universitario de Córdoba), hubo dos derechos que se pusieron en tensión: por un lado, el derecho de estudiantes a recibir educación, y, por otro lado, los derechos laborales de los y las trabajadoras docentes:

los sindicatos docentes tuvimos realmente que realizar una difícil tarea política que consistía en poder aunar, en hacer que no se contrapusieran distintos polos que estaban en tensión. Por un lado, uno de los que siempre cuesta... que son los derechos laborales, que por alguna mitología cultural los docentes y las docentes somos apóstoles de algo, somos mártires de alguna cosa. Siempre queda para el final discutir las condiciones de trabajo, y qué es lo que uno puede o no puede hacer en términos razonables. (CONVERSACIONES II, 2020b)

En este sentido, se pregunta una profesora: “¿Cómo garantizar el acceso a la educación a todos y cada uno de los estudiantes, sin que el exceso de los tiempos docentes regulado para la presencialidad se sienta como sobredemanda?" (CONVERSACIONES II, 2020b). Y también:

¿Qué hacemos con las gestiones que no alcanzan a divisar la necesidad de recuperar en la intencionalidad pedagógica, el trabajo en red con las organizaciones e instituciones barriales en las zonas vulneradas? ¿Hasta dónde el voluntarismo puro (y hasta sacro del oficio docente) va en contra de una postura políticamente comprometida con esa "nueva normalidad"? (CONVERSACIONES I, 2020C)

Un interrogante que se impone en ese escenario es: ¿qué hubiera pasado si los gremios docentes, frente a la falta de condiciones, hubieran promovido o consultado con quienes representan para no participar del despliegue educativo virtual de emergencia, hasta tanto no se garantizaran las condiciones mínimas para el trabajo? 
Lo cierto es que los convenios colectivos, estatutos y leyes que regulan la actividad docente de todos los niveles, por lo reciente de estos procesos (y en el caso de la pandemia, lo inesperado), presentan un vacío en este aspecto.

En el ciclo de Conversaciones, el Secretario General del gremio docente de Córdoba, Juan Monserrat, lo expresaba en estos términos: “Nuestras regulaciones, estatutos, nuestros cargos, los horarios de trabajo, los lugares de entrada y salida, los actos escolares, el salario, están organizados en función de una presencialidad. Ni siquiera imaginamos cómo puede ser el pago del salario de la virtualidad” (CONVERSACIONES II, 2020b).

Estos vacíos hicieron que en la tensión entre prestar el servicio educativo -con el compromiso que caracteriza al colectivo docente- las exigencias por parte de los niveles directivos, y la preservación de los derechos laborales, les docentes hayan elegido de manera generalizada responder a las expectativas de inclusión de la educación pública. Como lo expresaba otra profesora, "ningún docente se resistió a los cambios, sin legislación que nos avale" (CONVERSACIONES II, 2020b).

En este sentido, Monserrat señalaba también que una de las tareas o preocupaciones del gremio docente fue tratar

de que no haya una autoexplotación frente a las demandas de las comunidades, de los cuerpos directivos y supervisores que están permanentemente exigiendo a los compañeros que den más cuando están dando todo, y sin embargo pareciera que porque que no se ve el trabajo del docente se cree que no lo está haciendo [...] la autoexplotación es una forma continua y constante de ejercer la docencia. (CONVERSACIONES II, 2020b)

Fue en el marco de esos desafíos que se debatió en el Congreso de la Nación y se sancionó en agosto de 2020, la Ley 27.555 Régimen Legal del Contrato de Teletrabajo. La Ley, si bien no está orientada específicamente a la docencia, aborda aspectos por demás interesantes para pensar y repensar el trabajo docente. Por ejemplo, el Art. 9, referido a los elementos de trabajo, establece que es el empleador quien debe proporcionar el equipamiento necesario para que los trabajadores puedan desempeñar sus tareas (software y hardware), así como asumir los costos de instalación, mantenimiento y 
reparación de los mismos (ARGENTINA, 2020a, art. 9). Además, se estipula una compensación para las personas que utilicen sus propias herramientas para trabajar. En el Art. 10 se prevé que los gastos de conectividad y/o consumo de otros servicios deben ser compensados (ARGENTINA, 2020a, art. 10).

Tanto el uso de los propios equipos, conectividad y electricidad, como la falta de ellos en los propios domicilios, han sido objeto de denuncias y demandas recurrentes por parte de las y los docentes en el período de pandemia. Así lo expresaba una docente en Conversaciones: "Tampoco las gestiones han pensado cómo implementar democráticamente las tecnologías; no se consultó nada a los docentes sobre qué elementos tenían, si tenían o no wifi, qué saberes tecnológicos podían aportar o sugerir, formas de enseñanza colaborativas, etcétera" (CONVERSACIONES I, 2020c).

Otro aspecto que contempla la Ley es la instancia de la capacitación en el uso de tecnologías digitales. El Art. 11 señala que el empleador deberá garantizar la correcta capacitación de sus dependientes en nuevas tecnologías, lo cual no implicará una mayor carga de trabajo (ARGENTINA, 2020a, art.11). Esto, si bien se incluye en la ley para personas que trabajan bajo este régimen, para el caso de la educación ha sido siempre un aspecto problemático. En todos los niveles no solamente los y las docentes han asumido y asumen con sus propios salarios la formación y actualización que su cargo y su función requiere, sino que en aquellos casos en que la formación se ha brindado de manera gratuita, el tiempo/horas que insume esa capacitación no es paga, como sí lo es en otros rubros laborales. Esto es particularmente notable para la capacitación en el uso de tecnologías digitales. Es decir, se ha exigido y se exige a los profesores que sean innovadores en sus clases, para lo cual deben formarse por sus propios medios, en algunos casos realizando cursos pagos, y además hacerlo fuera del horario de trabajo por el cual se les paga. Son pocos los casos del universo laboral en los que la actualización en el uso de una nueva herramienta para el trabajo se haga por cuenta y riesgo de quienes trabajan. Algunas de estas cuestiones son abordadas al menos para lo que fueron las prácticas y las políticas de formación docente en el uso de TIC durante la década que acaba de finalizar, en Cabello y Morales (2011), Melendez; Diaz y Yuni (2017). 
Así fue expresado en nuestras Conversaciones: "El Estado Nacional y el Estado Provincial deben priorizar la investigación, seguimiento y formación de sus docentes. Por ahora todo lo hacemos Ixs docentes a pulmón y con el mismo o menor pago del servicio brindado" (CONVERSACIONES I, 2020C).

Incluso, tan perverso es el mecanismo, que se responsabiliza a las/los propios docentes por la falta de formación o por sus dificultades para el uso de las herramientas digitales: "La sobre carga de trabajo docente se ha profundizado durante la pandemia, y está acompañada de narrativas que atacan al sector público y a las y los trabajadores de la educación acusándoles de los supuestos "atrasos digitales" (OBSERVATORIO LATINOAMERICANO DE POLÍTICAS EDUCATIVAS, 2020a).

Sin embargo, y en el caso de la encuesta que realizamos, casi el 70\% del profesorado realizó alguna capacitación sobre el uso de TIC. Sin temor a equivocarnos, sea que tuvieron que pagarla o le fueron proporcionadas gratuitamente por el Estado, el tiempo que les insumieron esas capacitaciones fueron sustraídas a sus tiempos de descanso o de compartir con afectos. Además, más del $90 \%$ puedo aplicar esos conocimientos a su práctica docente, lo cual indica el compromiso y la creatividad con la propia tarea. El gráfico 4 expone claramente esta dimensión:

Gráfico 4: Capacitaciones en torno a lo digital y aplicación de conocimientos en la docencia

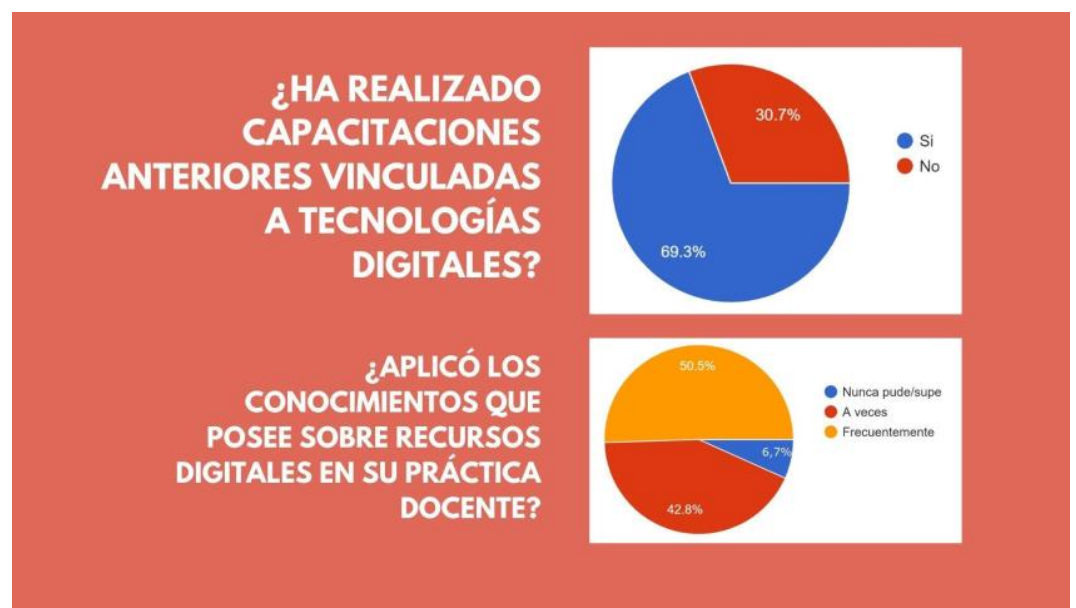

Fuente: Elaboración propia a partir de encuesta realizada a participantes del Ciclo Conversaciones en Red, 2020. 
En relación con las capacitaciones a las que les gustaría acceder, las que más fueron mencionadas por los docentes son, en orden decreciente:

1. Edición de video

2. Capacitación y actualización integral

3. Plataformas virtuales (Moodle, Classroom, etc.)

4. Herramientas colaborativas (Google Drive, Padlet, etc.)

5. Edición de sonido y producción de podcast

6. Herramientas y métodos de evaluación y seguimiento del aprendizaje

Otro aspecto de significativa importancia que aborda la Ley y que fue objeto de preocupación y justa queja por parte de docentes en pandemia, es el derecho a la desconexión. El Art. 5 de Ley expresa que

la persona que trabaja bajo la modalidad de teletrabajo tendrá derecho a no ser contactada y a desconectarse de los dispositivos digitales y/o tecnologías de la información y comunicación, fuera de su jornada laboral y durante los períodos de licencias. No podrá ser sancionada por hacer uso de este derecho. El empleador no podrá exigir a la persona que trabaja la realización de tareas, ni remitirle comunicaciones, por ningún medio, fuera de la jornada laboral. (ARGENTINA, 2020a, art. 5)

En el caso de la educación en pandemia, como dijimos, eso fue absolutamente imposible. Por un lado, porque las demandas estudiantiles ocurrían según sus propias posibilidades de conexión o ritmos específicos de la cultura juvenil. Por otro lado, ya existe una cultura en la actividad docente de trabajo en el hogar. Preparación de clases, corrección y evaluación, evaluaciones, formación, etc., ocurren fuera de los tiempos y horarios institucionales: en los hogares, solapando las dinámicas personales y familiares con la carga de trabajo. Si esto sucede normalmente, en condiciones de virtualidad de emergencia de la tarea educativa se agudizó. Habrá que pensar si esas son las mejores condiciones para afrontar una nueva etapa o habrá que exigir otras.

Por si fuera poco, a ello se suma el hecho de que la planificación, la producción de material y el monitoreo de las actividades educativas que incorporan TIC demandan muchísimo más tiempo que las que requieren otros insumos. Y, sin embargo, no son pagas. 
Esto es lo que también se ha puesto en evidencia durante la pandemia, para los propios trabajadores, para los y las estudiantes, para las familias, y, por supuesto, para dirigentes políticos y sindicales. "Los docentes estamos trabajando tres veces más que antes”, señala una docente en el Ciclo de Conversaciones, “perdiendo límites de horas de trabajo”, agrega otra, "estamos muy agobiados", concluye una tercera (CONVERSACIONES II, 2020b).

En julio de 2020 la Confederación de Trabajadores de la Educación (CTERA), publicó una encuesta denominada "Salud y condiciones de trabajo docente en tiempos de emergencia sanitaria COVID19", a la que respondieron más de 15.000 docentes de todo el país y de diversos niveles educativos, donde se señala que el $80 \%$ de las/os docentes dedican hoy más tiempo al trabajo que cuando hay clases presenciales. En parte, debido a “lo novedoso de la situación para docentes y estudiantes"; este dato da cuenta del proceso de aprendizajes que se está transitando, cuyo punto de partida es "una falta de formación generalizada para trabajar con esta modalidad" (CTERA, 2020).

Por otro lado, las actividades del trabajo que más tiempo les están demandando son, por un lado, la preparación de materiales didácticos; por otro lado, las comunicaciones con las/os estudiantes o sus familias, "lo cual muestra la complejidad y grado de dificultad que adquiere en este contexto esa dimensión del trabajo" (CTERA, 2020).

\section{Final abierto}

La educación en Argentina, como la pandemia, tiene un final abierto. No está claro qué modalidades asumirá el período de clases que en condiciones normales se inicia cada mes de marzo. Lo que sí está claro es que los procesos de enseñanza-aprendizaje seguirán ocurriendo de la mano de las tecnologías digitales.

Ello plantea desafíos, en primer lugar, para la política pública, que hasta el momento tiene dificultades para ofrecer a la ciudadanía -sobre todo a las comunidades educativasrespuestas diferentes a las de 2020. 
En segundo lugar, desafíos que se conectan con la adquisición de una visión global acerca de lo que significan las tecnologías digitales -cuya presencia se ha profundizado exponencial e inevitablemente en nuestra vida-.

No se trata de que algunos hagan negocios, eso ha sido históricamente así. Se trata, como dice Éric Sadin (2017), de la administración digital del mundo, de que paulatinamente transitamos hacia una delegación en unos sistemas informáticos de las decisiones que definen nuestra vida cotidiana, individual y colectiva. ¿Así lo hemos querido las personas comunes? ¿tenemos la libertad de decidir? No importa, es lo que efectivamente está ocurriendo. Por lo cual es necesario reconocer de qué modo sucede este debilitamiento en nuestras posibilidades de autonomía. Y, luego, aprender cómo podemos recuperar algo de esa autonomía. Eso es lo que sería deseable aprender como docentes -junto a los aprendizajes acerca del uso de aplicaciones y plataformas- para compartir con estudiantes. Como sostiene Jacob Appelbaum respecto del modo en que funcionan las computadoras,

a menudo la gente trata de sostener que tiene el derecho de poner eso bajo llave y mantenerlo en secreto, y hace computadoras complejas o hace que sea legalmente difícil comprenderlas. En realidad, eso es peligroso para la sociedad [...] la capacidad de acceder a los diagramas de los sistemas que subyacen a nuestras vidas es parte del motivo por el cual el software libre es importante, pero también de por qué el hardware libre es importante [...] ese es el motivo por el cual es importante comprender estos sistemas, porque cuando no los comprendemos, usualmente recurrimos a la autoridad, a quienes sí los comprenden o son capaces de ejercer control sobre ellos. (APPELBAUM, 2012 apud ASSANGE, 2012, p. 3637)

Tampoco se trata de plantear la discusión en términos de pesimistas u optimistas, de celebración o denostación. Son dicotomías improductivas para analizar el presente. Es necesario entender que el marco del debate es una disputa también social y política respecto de quién se apropia de los beneficios que el uso de tecnologías digitales genera, y con qué costo para trabajadores de la educación y para la sociedad en su conjunto. 


\section{Referências}

ARGENTINA. Ley Nacional N. 27.078 Argentina Digital, [Buenos Aires: Senado y Cámara de Diputados], 18 dic. 2014a. Disponible en:

http://servicios.infoleg.gob.ar/infolegInternet/anexos/235000-239999/239771/norma.htm. Acceso en: 11 dic. 2020.

ARGENTINA. Ministerio de Economía Nacional. Censo nacional del personal docente y no docente del sistema educativo argentino (CENPE). [Buenos Aires], 2014b. Disponible en: https://www.argentina.gob.ar/educacion/evaluacion-informacion-educativa/censos. Acceso en: 6 ene. 2021.

ARGENTINA. Ministerio de Economía Nacional. Presupuesto de la Administración Nacional: tercer trimestre. [Buenos Aires], 2016. Disponible en: http://docplayer.es/91523327-Presupuesto-de-la-administracion-nacional-tercertrimestre.html. Acceso en: 7 dic. 2020.

ARGENTINA. Ministerio de Educación y Deportes Nacional. Principales cifras del sistema educativo nacional. [Buenos Aires], 2017a. Disponible en:

http://www.bnm.me.gov.ar/giga1/documentos/EL005678.pdf . Acceso en: 6 ene. 2021.

ARGENTINA. Resolución 1536-E/2017, [Buenos Aires: Senado y Cámara de Diputados], 29 mar. 2017b. Disponible en: http://servicios.infoleg.gob.ar/infolegInternet/anexos/270000274999/273275/norma.htm. Acceso en: 11 dic. 2020.

ARGENTINA. Resolución 1410/2018, [Buenos Aires: Senado y Cámara de Diputados], 30 mayo 2018a. Disponible en:

https://www.argentina.gob.ar/normativa/nacional/resoluci\%C3\%B3n-1410-2018-311208 Acceso en: 11 dic. 2020.

ARGENTINA. Ministerio de Economía Nacional. Presupuesto de la Administración Nacional: cuarto trimestre. [Buenos Aires], 2018b. Disponible en: https://www.economia.gob.ar/onp/documentos/fisica_presupuesto/2018/4totrim18.pdf. Acceso en: 7 dic. 2020.

ARGENTINA. Ministerio de Economía Nacional. Presupuesto de la Administración Nacional: cuarto trimestre. [Buenos Aires], 2019. Disponible en: https://www.economia.gob.ar/onp/documentos/fisica_presupuesto/2019/4totrim19.pdf Acceso en: 6 enero 2021.

ARGENTINA. Ley Nacional 27.555. Régimen legal del contrato de teletrabajo. [Buenos Aires: Senado y Cámara de Diputados], 14 ago. 2020a. Disponible en: https://www.boletinoficial.gob.ar/detalleAviso/primera/233626/20200814. Acceso en: 11 dic. 2020.

ARGENTINA. Decreto 690/2020. [Buenos Aires: Senado y Cámara de Diputados], 21 de agosto de 2020b. Disponible en: 
https://www.boletinoficial.gob.ar/detalleAviso/primera/233932/20200822. Acceso en: 11 dic. 2020.

ASSANGE, Julian. Criptopunks. Barcelona: Deusto, 2013.

BARICCO, Alessandro. The game. Barcelona: Anagrama, 2019.

CABELLO, Roxana; MORALES, Susana. Enseñar con tecnologías: nuevas miradas en la formación docente. Buenos Aires: Prometeo: 2011.

CLAUS, Agustín; SANCHEZ, Belén. El financiamiento educativo en la Argentina: balance y desafíos de cara al cambio de década. Buenos Aires: CIPPEC, 2019. Disponible en: https://www.cippec.org/publicacion/el-financiamiento-educativo-en-la-argentina-balancey-desafios-de-cara-al-cambio-de-decada/. Acceso en: 27 dic. 2020.

CONVERSACIONES en Red I: la virtualización de la educación en contextos de vulnerabilidad educativa. Nancy Arias; Mariano Bilbao; Ivonne Henao Blasco; Lorena Asinari conversan con Alejandra Restagno. [S.I.]: Ciclo Conversaciones en Red, 4 jun. 2020a. 1 video (105 min.). Disponible en:

https://www.youtube.com/watch?v=LOMvoY6Gr5c.

Acceso en: 7 dic. 2020.

CONVERSACIONES en Red I: la virtualización de la educación en contextos de vulnerabilidad educativa. Yanina Arraya; Alejandra Restagno; Silvina Cuello conversan con Diego Moreiras. [S.I.]: Ciclo Conversaciones en Red, 9 jun. 2020b. 1 video (85 min.). Disponible en: https://www.youtube.com/watch?v=B-DbufPg7so. Acceso en: 7 dic. 2020

CONVERSACIONES en Red I: la virtualización de la educación en contextos de vulnerabilidad. Santiago Lucero y Alejandra Salgueiro conversan con Fabiana Castagno [S.I.]: Ciclo Conversaciones en Red, 11 jun. 2020c. 1 video (82 min.). Disponible en: https://www.youtube.com/watch?v=oSgPFHpIwLY. Acceso en: 7 dic. 2020.

CONVERSACIONES en Red I: la virtualización de la educación en contextos de vulnerabilidad. Estela Miranda y Enrique Bambozzi conversan con Laura Vargas. [S.I.]: Ciclo Conversaciones en Red, 18 jun. 2020d. 1 video (94 min.). Disponible en: https://www.youtube.com/watch?v=kGdzGxMmZ4g. Acceso en: 7 dic. 2020.

CONVERSACIONES en Red II: educación a distancia y virtual desde una perspectiva de derechos. Adriana Puiggrós; Adriana Fontana; Pablo Carro conversan con Susana Morales. [S.I.]: Ciclo Conversaciones en Red, 28 jul. 2020a. 1 vídeo (114 min). Disponible en: https://www.youtube.com/watch?v=8ce_hnO5BQc. Acceso en: 5 dic. 2020.

CONVERSACIONES en Red II: educación a distancia y virtual desde una perspectiva de derechos. Conversan Javier Blanco; Juan Monserrat; con Alejandra Restagno. [S.I.]: Ciclo Conversaciones en Red, 30 jul. 2020b. 1 vídeo (110 min). Disponible en: https://www.youtube.com/watch?v=n5pAPZrElhl. Acceso en: 05 dic. 2020. 
CONVERSACIONES en Red II: Educación a distancia y virtual desde una perspectiva de derechos. Conversan Nicolás Trotta; Yudith Rosenthal; Francisco Tamarit con Susana Morales. [S.I.]: Ciclo Conversaciones en Red, 13 ago. 2020c. 1 vídeo (109 min). Disponible en: https://www.youtube.com/watch?v=M2FIUwJV7Uo. Acceso en: 5 enero 2021.

CTERA. Informe de la Encuesta Nacional de CTERA: salud y condiciones de trabajo docente en tiempos de emergencia sanitaria COVID19. [Buenos Aires], 28 jul. 2020. Disponible en: https://www.ctera.org.ar/index.php/salud-laboral/publicaciones/item/3611informe-de-la-encuesta-ncional-de-ctera-salud-y-condiciones-de-trabajo-docente-entiempos-de-emergencia-sanitaria-covid19. Acceso en: 27 dic. 2020.

GT CLACSO. Apropiación de Tecnologías Digitales e Interseccionalidades y Riat. El acceso a las tecnologías digitales como derecho humano. Montevideo, mayo 2020. Disponible en: http://apropiaciondetecnologias.com/documentos/el-acceso-a-las-tecnologiasdigitales-como-derecho-humano/. Acceso en: 27 de dic. 2020.

LA GARGANTA PODEROSA: y después, nos dicen maleducados. [S.I.], 11 ago. 2020. Facebook: lapoderosa.ar. Disponible en: https://www.facebook.com/gargantapodero/posts/3250968674971973/. Acceso en: 19 dic. 2020.

LOS CAMBIOS en la educación argentina durante la pandemia de COVID-19: un estudio sobre la evolución de las prácticas escolares y familiares durante el aislamiento: segundo informe: cambios en la conectividad y uso de dispositivos tecnológicos para la educación durante el aislamiento. Observatorio Argentinos por la Educación, Buenos Aires, 2020. Disponible en:

https://cms.argentinosporlaeducacion.org/media/reports/ArgxEdu_Conectividad_Disposit ivos.pdf. Acceso en: 5 enero 2020.

MAURICIO Macri comparó la entrega de notebooks de Conectar Igualdad con "repartir asado". Diario Página 12, Buenos Aires, 23 mayo 2019. Disponible en:

https://www.pagina12.com.ar/195399-mauricio-macri-comparo-la-entrega-de-notebooksde-conectar-i. Acceso en: 5 dic. 2020.

MELENDEZ, C.E., DIAZ, A.G.; YUNI, J.A. Políticas de inclusión digital en la formación docente. Revista del Cisen Tramas, Maepova, v. 5, n. 2, p. 75-91, 2017. Disponible en: http://ppct.caicyt.gov.ar/index.php/cisen/article/view/10633. Acceso en: 19 dic. 2020.

\section{OBSERVATORIO LATINOAMERICANO DE POLÍTICAS EDUCATIVAS. EI comercio}

educativo: un hilo conductor pre y post pandemia. San José de Costa Rica, 15 jul. 2020 . Disponible en: http://www.observatorioeducacion.org/noticias/el-comercio-educativoun-hilo-conductor-pre-y-post-pandemia. Acceso en: 5 enero 2020.

OBSERVATORIO LATINOAMERICANO DE POLÍTICAS EDUCATIVAS. Soluciones digitales y tecnológicas públicas, estatales y gratuitas para detener el avance de transnacionales tecnológicas a la respuesta educativa. San José de Costa Rica, 15 sep. 2020b. Disponible en: http://www.observatorioeducacion.org/noticias/soluciones-digitales-y-tecnologicas- 
publicas-estatales-y-gratuitas-para-detener-el-avance. Acceso en: 5 enero 2020.

RODRÍGUEZ, Combertty (coord.). Tendencias en educación, San José de Costa Rica: Observatorio Latinoamericano de Políticas Educativas, 2017. Disponible en: https://ei-ieal.org/sites/default/files/docs/ie_tendencias02_web.pdf. Acceso en: 5 dic. 2020.

SADIN, Éric. La humanidad aumentada: la administración digital del mundo. Buenos Aires: Caja Negra, 2017.

SRNICEK, Nick. Capitalismo de plataformas. Buenos Aires: Caja Negra, 2018.

TRAS LOS despidos, Mauricio Macri ratificó la continuidad del Plan Conectar Igualdad. Diario La Nación, Buenos Aires, 10 mar. 2016. Disponible en: https://www.lanacion.com.ar/politica/tras-los-despidos-mauricio-macri-ratifico-lacontinuidad-del-plan-conectar-igualdad-nid1878472/. Acceso en: 19 dic. 2020.

TROTTA aseguró que buscarán al responsable por las 100 mil netbooks "abandonadas". Diario Perfil, Buenos Aires, 1 feb. 2020a. Disponible en: https://www.perfil.com/noticias/politica/nicolas-trotta-aseguro-buscaran-responsable-por100-mil-netbooks-abandonadas.phtml. Acceso en: 19 dic. 2020

UNESCO. Global education coalition. [S.I.], 2020. Disponible en: https://globaleducationcoalition.unesco.org/. Acceso en: 5 enero 2021.

VAIDHYANATHAN, Siva. La googlización de todo (y por qué deberíamos preocuparnos). Ciudad de México: Océano, 2012. 